\title{
Site Internet du génie de la conception mécanique
}

\author{
François Charron, ing. \\ Département de génie mécanique \\ Université de Sherbrooke \\ Francois.R.Charron@USherbrooke.ca
}

\section{Résumé}

Depuis 1992, le Département de génie mécanique de l'Université de Sherbrooke a introduit plusieurs nouvelles activités pédagogiques en conception. Évidemment, au cours de leur baccalauréat, les étudiants acquièrent plusieurs connaissances dans le domaine des mathématiques, des sciences fondamentales, des sciences du génie et des études complémentaires. Hors, la conception mécanique nécessite aussi des connaissances dans le domaine des techniques, des normes, des brevets, etc. Malheureusement, ces connaissances sont très peu enseignées dans les programmes de baccalauréat. C'est à la suite de ce constat que le site Internet du génie de la conception mécanique (www.conception.gme.usherb.ca) a été créé.

\section{Introduction}

La conception est au cœur de la pratique professionnelle de l'ingénieur. Évidemment, la conception requiert une bonne maîtrise du génie (corpus des connaissances scientifiques et techniques ${ }^{1}$ de l'ingénieur) et les sciences du génie sont le sujet principal des cours magistraux dans les programmes de baccalauréat. Par exemple, à la fin de sa formation, un ingénieur mécanique possède des connaissances dans le domaine des matériaux, de la résistance des matériaux, de la thermodynamique, de la fabrication mécanique, de l'aérodynamique etc. D’une manière similaire, les étudiants sont brièvement exposés aux principales techniques du génie mécanique. Toutefois, cette introduction qui est souvent et uniquement faite dans des leçons magistrales, ne permet pas aux étudiants d'atteindre un niveau de maîtrise adéquat. En

1 Technique: Ensemble d'objets ou de procédés pour concevoir et/ou produire une résultat déterminé (ex. : produit, machine, système, procédé de fabrication, etc.). Technologie: Étude des techniques, des outils, des machines, des matériaux, etc.

\author{
Jean-Philippe Desbiens, ing. jr. \\ Département de génie mécanique \\ Université de Sherbrooke \\ Jean-Philippe.Desbiens@,Usherbrooke.ca
}

somme, les diplômés en génie maîtrisent généralement bien les sciences du génie dans leur domaine de spécialisation (ex. : génie mécanique) mais ils sont toutefois relativement novice avec les techniques de l'ingénieur et plusieurs autres éléments importants de la pratique professionnelle de l'ingénieur comme les normes, les brevets, etc.

C'est une des raisons qui a motivé l'ajout de projets majeurs de conception au programme de génie mécanique de l'Université de Sherbrooke depuis 1992. Il s'avère que les projets majeurs de conception sont une bonne formule pédagogique favorisant, entre autres, l'apprentissage des techniques de l'ingénieur.

Cependant, peu d'outils efficaces sont disponibles pour aider les étudiants qui sont souvent des novices dans le domaine des techniques, à faire des choix judicieux de conception. Dans la pratique, l'ingénieur expert a recours à son expérience, à des bases de données et/ou à des catalogues pour sélectionner des pièces, des machines, des systèmes, etc. Ainsi, on retrouve souvent des bibliothèques techniques dans les entreprises. Cependant, les techniques évoluent rapidement et il s'avère souvent fastidieux de mettre à jour une telle bibliothèque pour suivre l'évolution des produits disponibles. La venue du Web (WWW) a permis de palier en partie à ce problème en amenant la création de sites Internet de fournisseurs complets et à jour. Depuis quelques années, il est même possible d'accéder par le Web à ces catalogues mais aussi à des outils de calcul, et même, de commander ces produits en ligne.

La recherche initiale de pièces ou de matériaux peut également s'effectuer dans des grands répertoires existant sur le Web (ex. : www.globalspec.com). Par contre, la quantité résultante d'informations accessibles est phénoménale au point où un étudiant ou un ingénieur novice peut difficilement identifier efficacement les grands fournisseurs et les ressources appropriées pour ses besoins. 
Dans le but d'améliorer l'apprentissage des techniques et de faciliter les choix de conception, un répertoire permettant de classer des informations sur différentes techniques a été développé. Concernant l'implantation, deux choix étaient possibles, soit de créer une bibliothèque technique dans un lieu physique (catalogues de fournisseurs, livres spécialisés dans le choix de composantes mécaniques, etc.) ou de développer un répertoire sur un site Internet contenant les principales références techniques couramment utilisées par les ingénieurs mécaniques. Évidement, les principaux désavantages de la bibliothèque technique étaient l'espace requis, la mise à jour continue des catalogues et des livres de référence, et la gestion quotidienne de la bibliothèque considérant que près de 200 étudiants travaillent sur leurs projets majeurs de conception lors d'une même session (automne). Pour toutes ces raisons, un répertoire électronique accessible via le Web à l'adresse www.conception.gme.usherb.ca a été mis sur pied.

Le présent article est divisé en trois parties. Premièrement, les principales activités pédagogiques associées avec les projets majeurs de conception réalisés par les étudiants sont présentées. Deuxièmement, les grandes lignes du corpus des connaissances et des techniques développé sont discutées. Finalement, le site Internet du génie de la conception est exposé.

\section{Les projets de conception}

Le baccalauréat en génie mécanique de l'Université de Sherbrooke se compose de 8 sessions d'étude, de 4 sessions de stages et d'une session libre. Dès la cinquième session (S5), les étudiants sont initiés au processus de développement de produit (PDP) et à la méthodologie de conception dans le cours "IMC156Méthodologie de la conception ". C'est également au cours de cette session que les étudiants forment leur équipe de projet (5-10 étudiants), qu'ils choisissent leur projet majeur de conception et qu'ils effectuent une pré-étude de faisabilité de leur projet. Lors de cette pré-étude, les étudiants sont amenés à effectuer une première recherche des techniques ayant un lien important avec leur projet. Cette recherche est très importante puisqu'elle permet de choisir certaines techniques considérant les contraintes du projet (ex. : budget, échéancier, etc.) et les compétences de l'équipe. Le mémoire d'identification de projet (MIP) présente les principaux résultats de cette pré-étude de faisabilité. Après l'approbation de la pré-étude, le projet se déroulera au cours des trois sessions subséquentes, soit une période de 16 à 20 mois.
Lors de la sixième session du programme (S6), la conception système est faite dans le cours "IMC900 Projet de conception $I »$. À la fin de ce cours, le rapport de conception système présentera entre autres, les besoins du client, les principales fonctions, les spécifications fonctionnelles et les concepts développés et retenus. À ce stade, les étudiants possèdent un cahier des charges fonctionnel, une structure fonctionnelle, une configuration physique et des concepts pour chacun des sous-systèmes.

Par la suite, la session S7 amène les étudiants à effectuer un dimensionnement préliminaire dans le cours "IMC906 - Projet de conception II». Des calculs préliminaires pour la sélection des techniques et le dimensionnement sont réalisés au cours de cette phase du PDP. Un prototype virtuel est aussi modélisé avec l'aide d'un logiciel de conception assistée par ordinateur $(\mathrm{CAO})$. Le rapport de conception est remis à la fin de la session et dans certains cas, la fabrication de prototypes simplifiés de certains sous-systèmes critiques peut être amorcée. Certaines composantes et/ou matériaux avec des temps de livraison importants sont commandés chez les fournisseurs à la fin de la session.

Ensuite, la conception du produit est raffinée dans le cadre du cours "IMC907 - Projet de conception III » en S8. Généralement, des calculs détaillés nécessitant des méthodes comme les éléments finis sont effectués pour vérifier le dimensionnement préliminaire. Parfois, la conception sera modifiée en raison de nouvelles contraintes ou des résultats provenant des analyses. C'est pourquoi, il y aura souvent des modifications de conception avant la fabrication complète du produit. Le prototype est fabriqué progressivement durant la session S8. Le projet se termine par une phase de validation qui suit la remise du rapport de conception détaillée. Le rapport de validation expérimentale (RVE) est rédigé. Il résume certains tests et mesures expérimentales effectuées sur le produit pour valider l'atteinte des spécifications fonctionnelles et techniques qui ont été définies au début du projet de conception. Des modifications au prototype sont alors proposées et selon le temps disponible, elles sont même implantées pour corriger les points faibles de la conception s'il y a lieu.

Évidemment, la réalisation d'un tel projet majeur de conception nécessite l'accès à un corpus des techniques de l'ingénieur mais aussi à des informations complémentaires à la formation académique comme les brevets et les normes. 


\section{Corpus des connaissances et des techniques}

La présente section présente le corpus des connaissances et des techniques développé, le classement des techniques retenues ainsi que la mise en œuvre du corpus.

\subsection{Corpus des connaissances}

Évidemment, la base du corpus des connaissances dans le domaine des mathématiques, des sciences fondamentales et des sciences du génie repose en grande partie sur les cours magistraux d'un programme de baccalauréat. Cependant, les étudiants ont souvent peu de connaissances dans les domaines suivants :

- Les propriétés et la mise en forme des matériaux (sélection, propriétés, mise en forme, fournisseurs);

- Les logiciels et outils de simulation numérique (ex. : $\mathrm{CAO} / \mathrm{IMAO} / \mathrm{FAO}$, MATLAB, SIMULINK, etc.);

- Les normes de l'industrie (ex.: ISO, SAE, ASME, etc.);

- Les brevets (ex. : OPIC, USPTO, etc.).

\subsection{Corpus des techniques}

Similairement au corpus sur les connaissances, celui des techniques fournit aux étudiants des informations sur différentes techniques du génie mécanique.

Ces principales techniques pour l'ingénieur mécanique peuvent se classer en deux grandes classes, soient :

- Les techniques de la mécanique traditionnelle (ex. : roulements, système de ventilation, moteurs à combustion, méthodes d'assemblage, etc.)

- Les techniques de la mécatronique (ex. : contrôle, programmation, électronique, etc.).

Bien entendu, la mécatronique est maintenant une branche incontournable en génie mécanique mais au cours des dernières années, on a constaté que les étudiants ont souvent des difficultés considérant la formation limitée qu'ils ont reçue et aussi le fait que certains aspects du génie électrique sont souvent moins intuitifs pour les mécaniciens.

\subsection{Classement des techniques}

Un des problèmes avec la mise en oeuvre d'un corpus est le classement des techniques retenues pour le développement d'un répertoire. Afin de développer un répertoire assez complet et facile à consulter, nous avons analysé plusieurs méthodes de classement des techniques (ex.: classement pour les brevets, classement dans des bibliothèques, classement dans des grands répertoires spécialisés sur le Web, etc.). À la suite de l'analyse de ces différentes méthodes de classement, nous avons développé les classes suivantes pour notre répertoire :

- A. Assemblage

$\begin{array}{cl}\checkmark & \text { Adhésif } \\ \checkmark & \text { Attaches non filetées } \\ \checkmark & \text { Attaches spéciales } \\ \checkmark & \text { Boulons } \\ \checkmark & \text { Écrous } \\ \checkmark & \text { Joints }\end{array}$

- B. Chauffage et climatisation

$\checkmark$ Chauffage et ventilation

$\checkmark \quad$ Échangeurs de chaleur

$\checkmark$ Réfrigération ou refroidissement

- C. Éléments mécaniques

$\begin{array}{cl}\checkmark & \text { Accouplements } \\ \checkmark & \text { Arbres } \\ \checkmark & \text { Câbles } \\ \checkmark & \text { Chaînes } \\ \checkmark & \text { Courroies } \\ \checkmark & \text { Embrayages } \\ \checkmark & \text { Engrenages } \\ \checkmark & \text { Entraînements à vitesse fixe } \\ \checkmark & \text { Entraînement à vitesse variable } \\ \checkmark & \text { Freins } \\ \checkmark & \text { Ressorts } \\ \checkmark & \text { Roulements } \\ \checkmark & \text { Composantes mécaniques de précision }\end{array}$

- D. Hydraulique et pneumatique

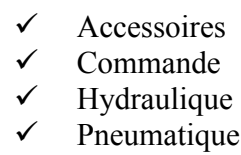

- E. Matériaux

$\begin{array}{cl}\checkmark & \text { Autres matériaux } \\ \checkmark & \text { Composites } \\ \checkmark & \text { Élastomères } \\ \checkmark & \text { Métaux ferreux } \\ \checkmark & \text { Métaux non ferreux } \\ \checkmark & \text { Plastiques }\end{array}$

- F. Mécatronique
$\checkmark$ Actionneurs
$\checkmark$ Capteurs
$\checkmark \quad$ Circuits électriques de base
$\checkmark$ Composantes électriques de base
$\checkmark$ Contrôleurs

- G. Moteurs à combustion 
$\checkmark \quad$ Allumage par bougie

$\checkmark$ Diesel

$\checkmark \quad$ Turbine à gaz

\section{- H. Moteurs électriques}

$\checkmark$ Contrôleurs

$\checkmark$ Moteurs AC

$\checkmark$ Moteurs DC

$\checkmark$ Moteurs linéaires

$\checkmark \quad$ Moteurs pas à pas

$\checkmark$ Servomoteurs

$\checkmark$ Applications spéciales

Après une évaluation préliminaire, il s'avère que ce classement relativement simple permet vraisemblablement de couvrir une grande majorité des besoins initialement identifiés.

\section{Site Internet du génie de la conception}

\subsection{Répertoire ou outil de recherche}

Avant de réaliser l'implantation, une réflexion a été faite sur la question de la création d'un outil de recherche par mots clés (ex.: Google) ou d'un répertoire avec des classes (ex. : Yahoo).

L'efficacité et l'exactitude de la recherche par mots clés reposent essentiellement sur la qualité des termes liés aux techniques du génie mécanique. De toute évidence, un minimum de connaissances concernant les techniques est nécessaire pour réaliser des recherches efficaces.

D'autre part, les répertoires ont l'avantage de proposer des classes précises avec plusieurs niveaux permettant de réaliser des recherches hiérarchiques (ex. : d'une classe à une information détaillée sur un distributeur). L'avantage de cette approche est qu'un novice peut identifier facilement les principales techniques disponibles ainsi que les grands fournisseurs de cellesci sur le marché. Le principal désavantage est qu'un répertoire est toujours incomplet et qu'un expert ne trouvera pas toutes les informations pertinentes à son projet. En contrepartie, l'expert a accès à d'autres outils de recherche disponibles sur le Web. Nous avons donc retenu l'approche d'un répertoire car elle est décidément plus adaptée aux besoins des étudiants en génie qui sont souvent des novices en conception.

Bien entendu, notre objectif n'était pas d'inclure toutes les techniques disponibles sur le marché ainsi que les coordonnées de tous les fournisseurs. C'est pourquoi nous avons ciblé les classes mentionnées précédemment. Ces dernières couvrent la majorité des recherches effectuées par nos étudiants. Pour les éléments plus spécialisés, les étudiants ont la responsabilité de dénicher eux-mêmes l'information.

\subsection{Implantation du corpus des connaissances et des techniques}

Le cœur du site Internet du génie de la conception est constitué d'une section intitulée «Ressources pour les projets » où on retrouve le corpus des connaissances et des techniques en génie mécanique.

Le corpus des techniques a été classé dans une soussection du site Internet intitulée « Distributeurs ». Il est possible d'accéder à l'information en naviguant dans les sous-menus des différentes classes du répertoire. À titre d'exemple, la Figure 1 présente le résultat d'une recherche sur les roulements à billes où on retrouve les principaux fabricants de roulements, les coordonnées des distributeurs et des liens hypertextes vers le site corporatif de la compagnie SKF, par exemple.

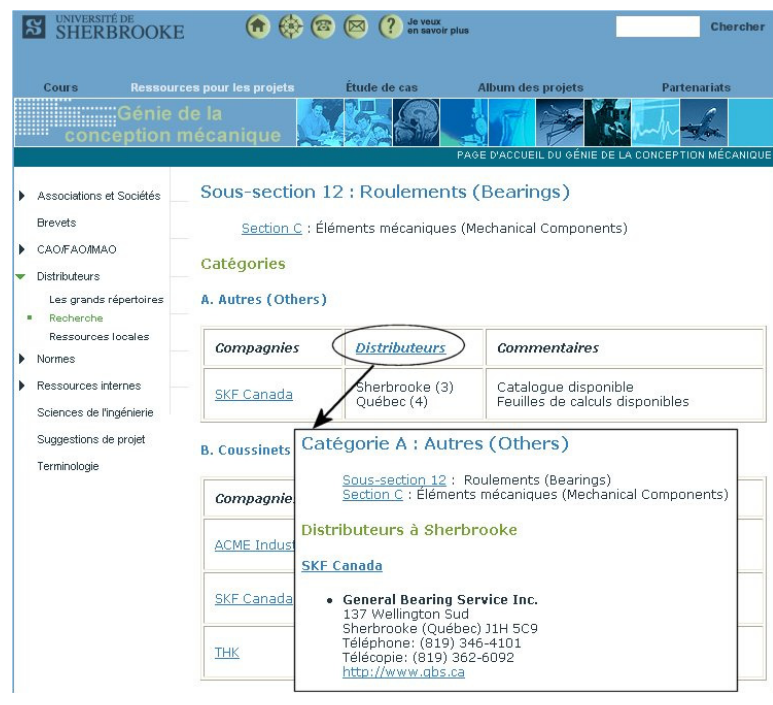

Figure 1. Recherche sur les roulements à billes dans le corpus des techniques

La plupart des fournisseurs importants comme la compagnie SKF possèdent des sites Internet complets incluant les éléments suivants :

- des catalogues en ligne;

- des outils de calculs et de sélection de composantes;

- des bibliothèques de pièces virtuelles téléchargeables;

- des outils de commande des produits en ligne. 


\subsection{Autres sections du site Internet}

Le site Internet du génie de la conception n'est pas seulement dédié aux corpus des connaissances et des techniques. En effet, ce site Internet accessible au grand public est également utilisé aux fins suivantes.

\subsubsection{Cours}

La section «Cours» permet la diffusion d'informations à propos des cours en conception (IMC156, IMC900, IMC906 et IMC907). De plus, le site Internet permet de faciliter la communication entre le corps professoral et les étudiants des projets de conception.

\subsection{2 Étude de cas}

La section «Étude de cas » contient des documents de référence pour l'enseignement des cours en conception. Par exemple, la documentation technique complète d'un projet de conception majeur appelé « $\mathrm{McGrO}$ » est incluse. Cette section contient également des outils de rédaction et des modèles de dessins techniques faits selon les règles de l'art [1].

\subsubsection{Album}

Cette section est consacrée aux projets de conception réalisés au cours des six dernières années au Département de génie mécanique. Près d'une soixantaine de projets (57) sont décrits pour informer et inspirer les futurs étudiants.

\subsubsection{Partenariats}

La section «Partenariat» informe les entreprises de la possibilité de parrainer un projet majeur de conception. Les modalités et les obligations d'un tel partenariat sont clairement expliquées via le guide de l'entreprise disponible en format électronique sur le site.

\section{Conclusion}

Le Département de génie mécanique de l'Université de Sherbrooke a introduit des projets de conception majeurs dans son programme de baccalauréat depuis 1992. Suite à plusieurs années d'expérience dans l'utilisation des projets de conception, des constats importants ont été faits.

Les cours magistraux permettent aux étudiants d'acquérir les connaissances dans les domaines des mathématiques, des sciences fondamentales, des sciences du génie et des études complémentaires. Toutefois, certaines notions comme la sélection des techniques, le choix des matériaux, la fabrication mécanique, les normes et les brevets sont rapidement couverts en classe et se prêtent d'ailleurs souvent très mal à cette formule pédagogique. Le niveau de maîtrise des techniques est par conséquent très faible à moins qu'elles soient mises en application dans des cas concrets. Ceci est d'ailleurs une des raisons d'être des projets de conception car ils permettent le développement de compétences essentielles à la pratique du génie.

Ainsi, dans le but de faciliter l'apprentissage des techniques et de connaissances complémentaires comme les normes et les brevets, un site Internet du génie de la conception mécanique fut développé afin de répertorier l'ensemble de ces ressources. Le fait d'utiliser le Web comme média permet l'accès à ces informations à l'ensemble de nos étudiants.

Bien que le concept d'un répertoire ait été préféré à celui d'un outil de recherche par mots clés pour constituer notre corpus des techniques, il n'est pas impossible que cette dernière fonctionnalité soit ajoutée pour les utilisateurs plus expérimentés dans le futur.

De toute évidence, des mises à jour seront essentielles pour que le site Internet reflète l'évolution du marché. Les étudiants sont d'ailleurs invités à contribuer dans ce sens. D'autres informations sont également disponibles sur le site Internet tel qu'un exemple de projet de conception majeur documenté ainsi qu'un album de tous les projets réalisés jusqu'à ce jour au Département.

Finalement, nous sommes convaincus que l'ajout de cet outil innovateur ${ }^{2}$ augmentera grandement l'efficacité de nos étudiants lors de la réalisation de projets de conception ainsi que leur connaissance générale et leur maîtrise des techniques du génie mécanique.

\section{RÉFÉRENCES}

[1] Charron, F. et Desbiens, J.P., Documentation d'un projet de conception, CDEN Design Conference, Montréal, Canada, 2004.

\footnotetext{
2 Pour plus de détails, visitez le site Internet à
} www.conception.gme.usherb.ca. 